\title{
CORRIGENDUM
}

\section{B-cell lymphoma/leukemia 10 promotes oral cancer progression through STAT1/ATF4/S100P signaling pathway}

T-S Wu ${ }^{1}$, C-T Tan ${ }^{2}$, C-C Chang ${ }^{3}$, B-R Lin ${ }^{4}$, W-T Lai ${ }^{1}$, S-T Chen ${ }^{5,6}$, M Yen-Ping Kuo ${ }^{1}$, C-L Rau ${ }^{7,8}$, F-S Jaw ${ }^{7}$ and H-H Chang ${ }^{1,9}$

Oncogene (2017) 36, 5440; doi:10.1038/onc.2017.250; published online 17 July 2017

Correction to: Oncogene (2015) 34, 1207-1219; doi:10.1038/onc. 2014.43; published online 31 March 2014

Since the publication of the above article, the authors have noticed that the stable transfectant named 'SAS/shBCL10/ S100P\#5' in Figure $5 \mathrm{~d}$ was mislabeled. The correct label should be 'SAS/shBCL10/S100P\#4'.

The authors have also noticed that the author affiliations were incorrect. The correct affiliations can be found below:

${ }^{1}$ Graduate Institute of Clinical Dentistry, School of Dentistry, National Taiwan University, Taipei, Taiwan;

${ }^{2}$ Department of Otolaryngology, National Taiwan University Hospital, Taipei, Taiwan;

${ }^{3}$ Graduate Institute of Oral Biology, School of Dentistry, National Taiwan University, Taipei, Taiwan;

${ }^{4}$ Department of Surgery, National Taiwan University Hospital, Taipei, Taiwan;
${ }^{5}$ Department of Pediatrics, National Taiwan University Hospital, Taipei, Taiwan;

${ }^{6}$ Department of Pediatrics, National Taiwan University Hospital YunLin Branch, YunLin, Taiwan;

${ }^{7}$ Institute of Biomedical Engineering, National Taiwan University, Taipei, Taiwan;

${ }^{8}$ Department of Physical Medicine and Rehabilitation, Shuang Ho Hospital, Taipei Medical University, Taipei, Taiwan and

${ }^{9}$ Department of Dentistry, School of Dentistry, National Taiwan University, Taipei, Taiwan

The authors would like to apologise for these errors and the inconvenience this may have caused. 